\title{
A Compact UWB Antenna Array for Breast Cancer Imaging Application using Optimisation Algorithm
}

\author{
Isah Danjuma $^{1}$, Allan Mertolla ${ }^{1}$, Ali Alabdullah ${ }^{1}$, Buhari Muhammad $^{1}$, Abdalfettah asharaa $^{1}$ \\ and Raed Abd-Alhameed ${ }^{1,2}$ \\ \{issamusa2001@yahoo.co.uk,a.mertola@bradford.ac.uk,a.a.alabdullah1@bradford.ac.uk,m.b.abubakar1 \\ @ student.bradford.ac.uk,a.s.m.asharaa@bradford.ac.uk and r.a.a.abd@bradford.ac.uk\} \\ ${ }^{1}$ School of Electrical Engineering and Computer Science, University of Bradford, Bradford \\ BD71DP, United Kingdom \\ ${ }^{2}$ Information and Communication Eng. Department, Basrah University College of Science and \\ Technology, Basrah 24001, Iraq
}

\begin{abstract}
A low profile ultra-wide band (UWB) antenna array is presented for breast cancer detections using microwave imaging experiments. The sensor was designed to operate in the UWB band frequency scale of 3.1-10.6 GHz. Simulated and calculated result of the sensor are in good agreement. A dielectric with plastic material having low electrical properties was used to support the array elements experiments. The array configuration has a good mutual coupling between the adjacent antennas of below -20dB.
\end{abstract}

Keywords: antenna optimisation, breast cancer detection, monopole antenna, array of antenna.

\section{Introduction}

Recently, ultra-wideband microwave imaging application have gained a lot of attention due to their intrinsic characteristics of small power consumption, low cost and high data transmission rate [1]. This application also finds current relevance for numerous medical applications including breast cancer assessment [2]. The manifestation of breast lump occur as a result of tumor inside the breast tissue and this was reported as one of the main causes of death among women globally [3]. Detection of breast cancer in its primary stage is the best option for its treatment. This underlines the serious need for a very reliable and comfortable method of breast tumor detection to serve as a complementary method to the currently used technology such as ultrasonography, imaging using magnetic resonance, computerized tomography and x-ray mammography [4].

Sensor using electromagnetic waves provides many advantages for tumour detection including non-ionizing and non-destructive testing. Other are low cost, low complexity and high data rate void of breast compression as compare to x-ray mammography. An antenna is usually used as the main sensor for imaging in the transmission and reception of microwave signals into 
the breast. This idea depends on the fluctuation of the dielectric properties of the breast configuration including relative permittivity and conductivity. The resultant signal reflected from the sensor are utilised to detects the difference in the electrical properties between normal and cancerous tissue in a more cost-effective, harmless and suitable manner. This technique functions with the variance of the water properties to differentiate normal tissue from the abnormal tissue in detecting the tumor. Healthier cells are perceptible to electromagnetic wave in comparison to unhealthy ones.

Percentage with higher water ratio within the cancerous tissues serves as the determinant for the tumor detection [4]. Thus, imaging using microwaves antenna is another alternative for early breast cancer detection, thereby saving a lot of life. Numerous UWB sensors were used in the past for imaging using array measurements, such as bow tie and Vivaldi antenna [5, 6]. The use of monopole antenna for the microwave-imaging array offers a very effective and performance particularly in the near field of the breast phantom [7]. Antenna fundamentals including return loss, VSWR, and radiating pattern over the UWB spectrum are well characterized.

In this work, a new, low cost with an enhanced impedance bandwidth and high-performance monopole antenna for breast cancer microwave imaging is presented. The antenna is small and uniform in size suitable for the microwave imaging array application. In this study, four array configurations were analysed and the simulation results indicate good signal transmission with low mutual coupling. The subsequent part of this work are as follows: Section II entails the antenna geometry including optimization design, Section III analyses the antenna performance measurements and Section IV concludes this work.

\section{Antenna Geometry and Optimisation Design}

The design geometry of an antenna is a very difficult and challenging task that need to be taken into consideration particularly when demanding an antenna with fundamental properties including large bandwidth, impedance matching and radiation pattern across the UWB range of the spectrum of interest.

\subsection{Antenna Geometry}

The proposed antenna has a uniform geometry of $30 \times 30 \mathrm{~mm}^{2}$ placed on $1.6 \mathrm{~mm}$ thickness, permittivity of 4.6, and loss tangent of 0.0025 FR-4 substrate. The sensor composition consists of a hexagonal patch with a circular slot, a feed line with a pair of uniform rectangular planes serving as an incomplete ground. The hexagonal patch parameters were as follows: Substrate length (SL), Substrate width (SW), Patch length (PL) Microstrip length (ML), Microstrip width (MW), short side (SS), Long side (LS), radius (R), and angle $\left(\mathrm{a}^{0}\right)$, as shown in Fig.1 and Fig. 2 
respectively. The values of UWB antenna parameters are tabulated in Table1. These parametric quantity were optimized to achieve the best result used for the microwave imaging array and the antenna is fed with $50 \Omega$ coaxial transmission line.

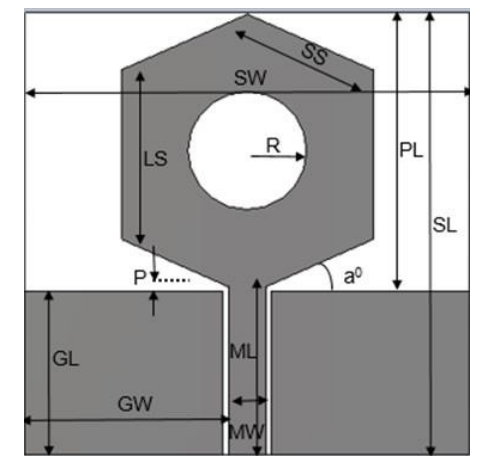

Fig.1. Layout geometry of the hexagonal monopole antenna.

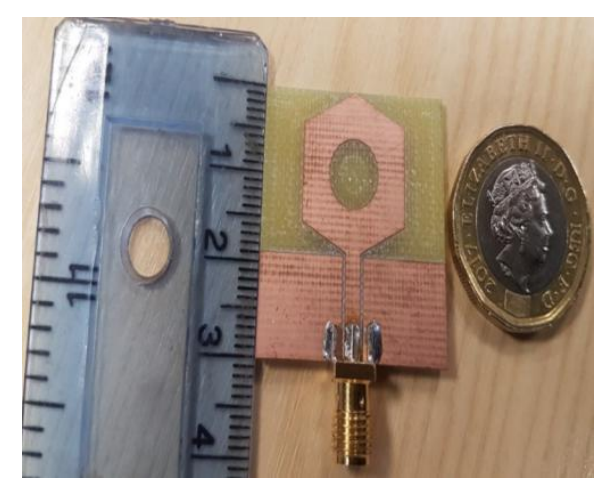

Fig.2. Physical implementation of the hexagonal monopole. 


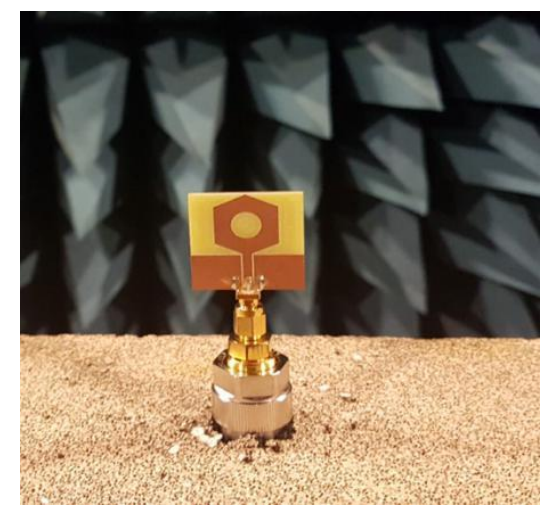

Fig.3. Far field Measurement of the proposed hexagonal monopole antenna.

Table 1. Dimensions specification of the proposed sensor.

\begin{tabular}{|l|l|}
\hline Parameter & Dimensions in mm \\
\hline Substrate width (SW) & 30 \\
\hline Substrate length (SL) & 30 \\
\hline $\begin{array}{l}\text { Substrate thickness } \\
\text { (ST) }\end{array}$ & 1.6 \\
\hline Microstrip length(ML) & 11.40 \\
\hline $\begin{array}{l}\text { Microstrip width } \\
\text { (MW) }\end{array}$ & 2.50 \\
\hline Microstrip gap (MG) & 0.4 \\
\hline Long Side (LS) & 11.49 \\
\hline Short Side (SS) & 9.30 \\
\hline Radius (R) & 4 \\
\hline Angle (a $\left.{ }^{0}\right)$ & 25 \\
\hline $\begin{array}{l}\text { Partial ground } \\
\text { length(GL) } \\
\text { Partial ground width } \\
\text { (GW) }\end{array}$ & 11.10 \\
\hline
\end{tabular}




\subsection{Optimisation Design}

Optimization is usually required in every antenna designs to enable designer to acquire the optimum results from their parametrise quantities. A little changes in the design specifications can have a significant variations on the system performance. Thus; in a system having different composition, their design fundamentals can be very difficult and therefore to acquire their best value can be impossible. The computer simulation technology (CST) software is used for the design and optimisation by using the trust region frame work of the CST optimisation algorithm. The algorithm take the benefit of S-parameter attributes to decrease the performance evaluations of the optimization process [8]. This serve as the most important part of the optimization algorithms. The factors that affect the efficient design of the proposed monopole UWB sensor are the gap between the pair uniforms rectangular planes serving as the partial ground, the partial ground length (GL and the angle at the bend of the corners of the ground plane $\left(\mathrm{a}^{0}\right)$. These parameters affects the proposed antenna radiation pattern, return loss and the impedance matching and hence, optimised to achieve better antenna performance as indicated in the return loss of Fig. 4 and 5 respectively.

\section{Antenna Performance Measurement}

The results of the simulated sensor were analysed and optimized with the aid of CST microwave studio. The simulated results was plotted using mat lab software while the measured results were obtained using Agilent technologies N5242. A vector network analyser cover the spectrum ranging $10 \mathrm{MHz}-26 \mathrm{GHz}$. The far-field measurement is in Fig. 3.The simulated measurement is shown in Fig.4, while both the simulated and calculated return loss results are indicated in Fig.5. It is obvious proposed antenna covers the UWB spectrum ranging from 3.1 to $10.6 \mathrm{GHz}$ with $-10 \mathrm{~dB}$.

The first resonance is indicated at $3.99 \mathrm{GHz}$, the second resonance at $6.5 \mathrm{GHz}$ while the highest resonance is at $10.6 \mathrm{GHz}$. The lower frequency bandwidth is largely affected by the use of the slot in the patch of the monopole antenna. The current flow of the design sensor is usually achieved by slot etching on the radiating patch. This development affects the surface current distribution as shown in Fig. 6. The radiation performance of the antenna in Fig.7, can then be controlled by examining the path of the surface current [9]. Although, the proposed sensor design is plain and compact in size, it achieves the overall impedance bandwidth of the UWB spectrum and the simulated and calculated results are in good matching.

The antenna array configuration was analysed having each antenna element arranged half way from each other with $360 / \mathrm{n}$ where $\mathrm{n}$ represent the number of the antenna elements. The configuration of circular array design was used for this experiment to model the three-layer 
phantom consisting of tumor, fat and skin. The tumor has a dimension of radius $4 \mathrm{~mm}$ and the scattering material used to model the tumor consisted of $10 \mathrm{~g}$ of wheat flour mixed with $6.5 \mathrm{~g}$ of water acting as a solvent. The tumor mixture electrical parameters has a relative permittivity of 25 and a conductivity of $2.59 \mathrm{~S} / \mathrm{m}$ at a spectrum of $4.5 \mathrm{GHz}$ [10]. This substance is used to present tumors of different sizes and usually used within the breast phantom for laboratory purposes.

The breast sample electrical properties were taken from [11] and the modelling of skin and fat phantom were taken from CST's material library. The skin and fat have the following dimensions: skin with radius $82 \mathrm{~mm}$, thickness of $2 \mathrm{~mm}$ and depth of $32 \mathrm{~mm}$, fat with radius $80 \mathrm{~mm}$ and depth of $32 \mathrm{~mm}$. The plastic layer was modelled around the breast phantom as it was used in [12] to support the antenna array. The plastic layer has a dimension including relative permittivity of $\varepsilon_{\mathrm{r}}=3$, conductivity of $\sigma=0.0015 \mathrm{~s} / \mathrm{m}$, radius $=92 \mathrm{~mm}$, thickness $=8$ $\mathrm{mm}$ and depth of $32 \mathrm{~mm}$. In order to compare the performances of the experiments, four antenna array configurations were conducted. The breast phantom and four-antenna array elements configuration are indicated in Fig. 8. This particular array configuration was chosen as the design is proposed for a hemispherical cup having radius of $101 \mathrm{~mm}$ adopting a rotation mechanism for the microwave breast imaging application. The coupling between the arrays elements with the breast phantom is shown in Fig. 9. There is good mutual coupling between the corresponding antennas, which is below $-20 \mathrm{~dB}$.

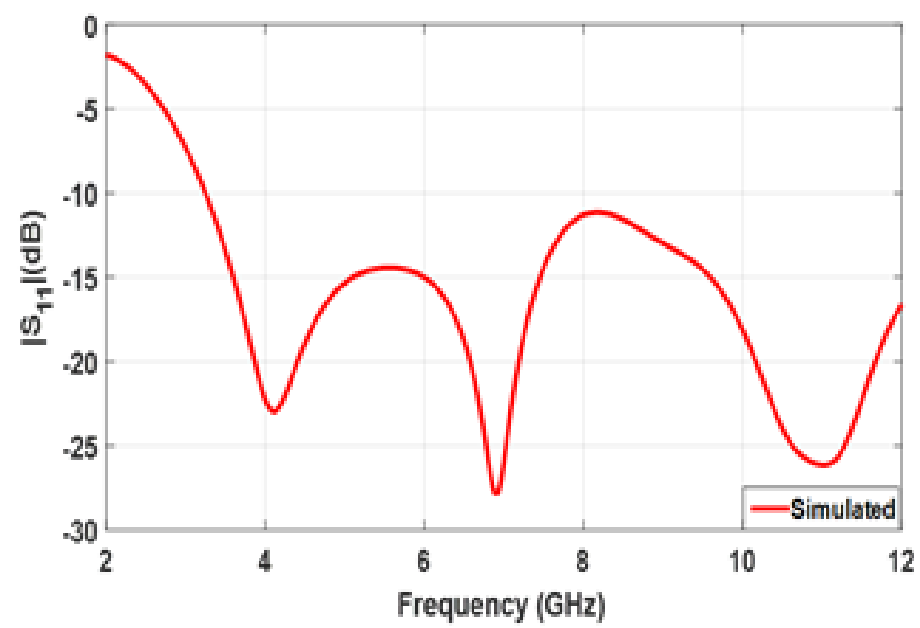

Fig.4. Simulated results with optimized parameters of gap 4mm and partial ground length GL $=11.10$ $\mathrm{mm}$ and angle of $25 .{ }^{\circ}$ 


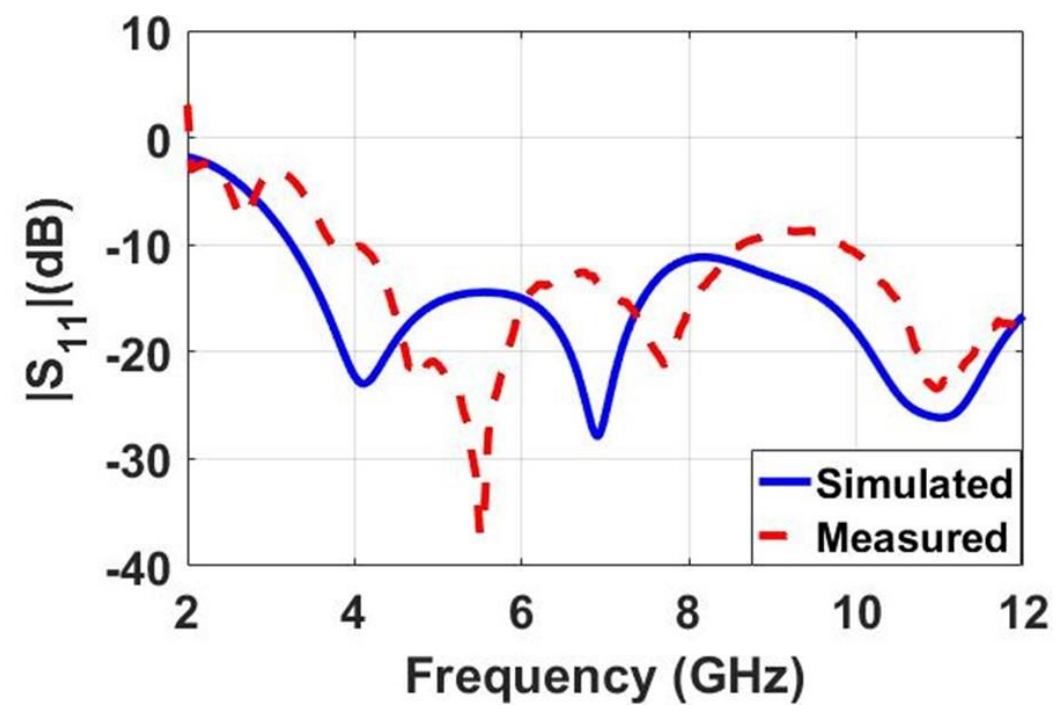

Fig. 5. Simulated and calculated results of the proposed antenna.
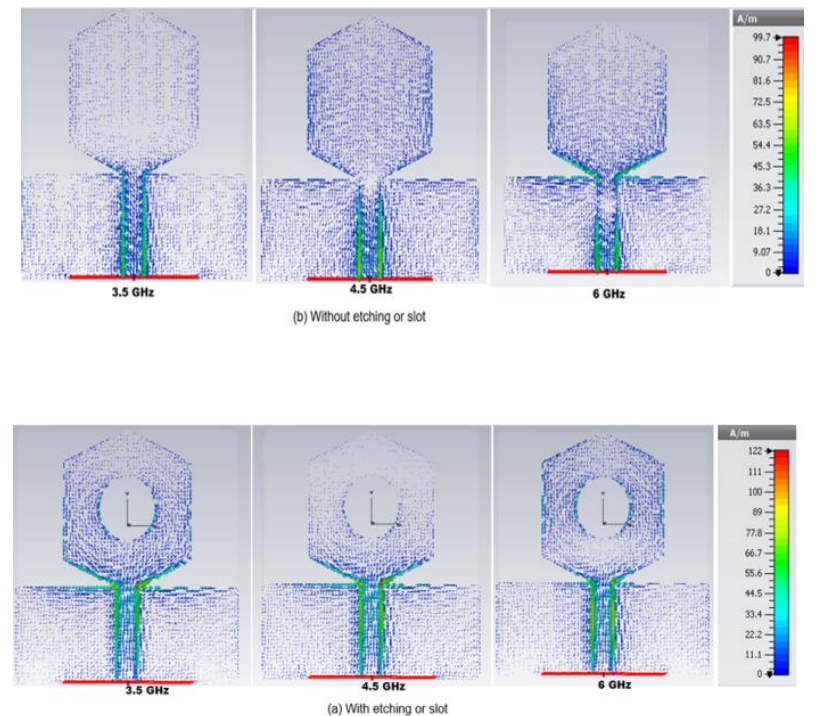

Fig.6. Surface current densities of the proposed antenna. 


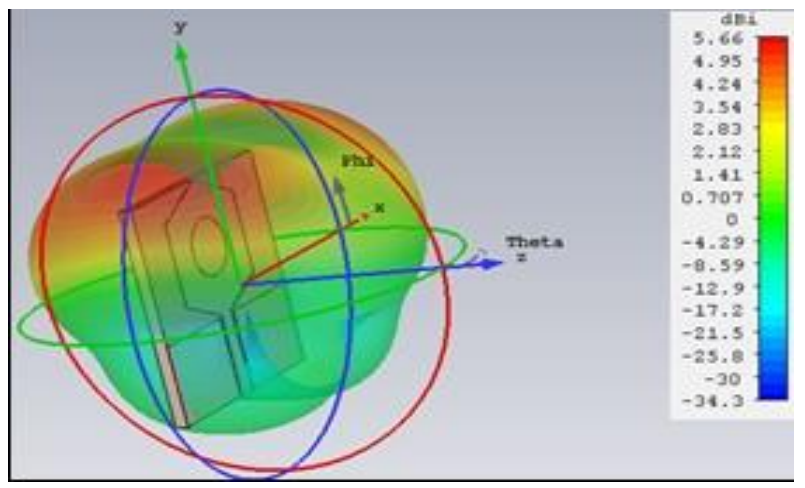

Fig.7. 3D far-field radiation pattern of the proposed hexagonal monopole sensor.

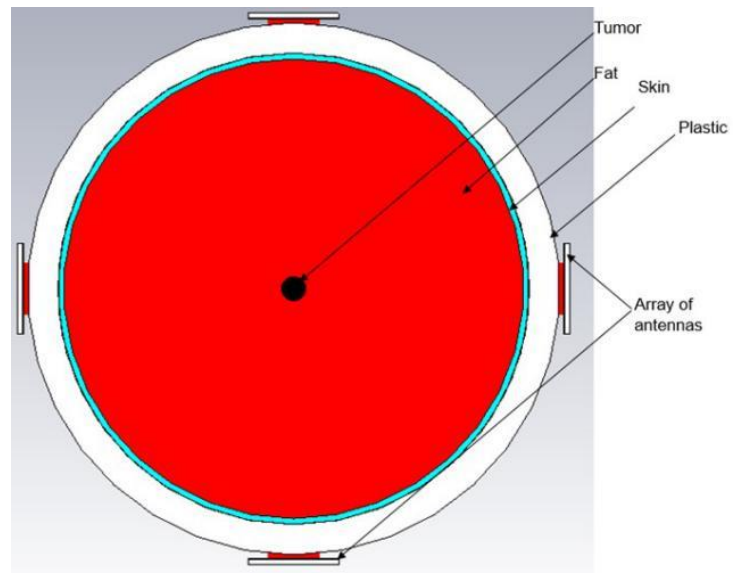

Fig.8. Model of the phantom with the four antenna array elements. 


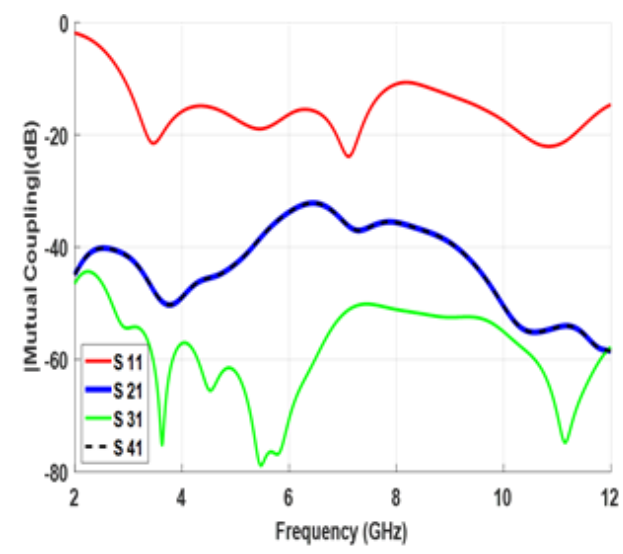

Fig.9. Mutual coupling of the $4 \times 4$-array element of the antenna.

\section{Conclusion}

In this a paper, an experimental analysis using a compact UWB sensor array for breast tumor imaging using electromagnetic wave has been presented. The low profile and the simple design of the antenna make it a better sensor for microwave imaging tasks. The antenna performance is measured based on the good mutual coupling between the corresponding antenna array elements. The antenna uniform size of $30 \times 30 \mathrm{~mm}^{2}$ is good enough for integration into many microwave devices.

\section{References}

[1] Haraz, O. and A.-R.: Sebak, p. 125-152.UWB antennas for wireless applications. Advancement in microstrip antennas with recent applications, (2013)

[2] Adnan, S.: Simulation and experimental measurements for near field imaging. Progress In Electromagnetics Research, (2012)

[3] Parkin, D.M.: Estimating the world cancer burden: Globocan 2000. International journal of cancer, p. 153-156 (2001)

[4] Danjuma, I.M.: AT slot monopole antenna for UWB microwave imaging applications, (2018) 
[5] Danjuma, I.M.: Design and optimization of a bowtie dipole adjacent to dielectric material for through the wall imaging using hybrid electromagnetic computational analysis techniques. in Internet Technologies and Applications (ITA), (2017)

[6] Danjuma, I.M.: Microwave imaging using arrays of Vivaldi antenna for breast cancer applications p.32-37 (2018)

[7] Danjuma, I.M.: A Compact size and low profile Rectanular slot monopole antenna for UWB body centric applications. (2019)

[8] Liu, B.: Efficient global optimisation of microwave antennas based on a parallel surrogate modelassisted evolutionary algorithm. IET Microwaves, Antennas \& Propagation, (2018)

[9] Yaghjian, A..: Equivalence of surface current and aperture field integrations for reflector antennas. IEEE Transactions on antennas and propagation, p. 1355-1358 (1984)

[10] Mirza, A.F.: An active microwave sensor for near field imaging. IEEE Sensors Journal,p. 27492757 (2017)

[11] Danjuma, I.: Design of a taper slot low profile Vivaldi antenna for ultra-wideband microwave breast imaging applications. Journal of Multidisciplinary Engineering Science and Technology (JMEST) (2018).

[12] Ahsan, S.: Balanced antipodal vivaldi antenna for microwave tomography. in 2014 4th International Conference on Wireless Mobile Communication and Healthcare-Transforming Healthcare Through Innovations in Mobile and Wireless Technologies (MOBIHEALTH). (2014) 